\title{
AVALIAÇÃO DA ATIVIDADE TRIPANOCIDA, LEISHMANICIDA E CITOTÓXICA DO GERANIOL E CITRONELAL
}

Joara Nalyda Pereira Carneiro $^{1}$; Rosimeire Sabino Albuquerque ${ }^{1}$; Nádghia Figueiredo Leite ${ }^{1}$; Antonio Júdson Targino Machado ${ }^{1}$; Dara Isabel Vieira de Brito ${ }^{1}$; Miriam Rolón ${ }^{2}$; Celeste Vega ${ }^{2}$; Cathia Coronel $^{2}$; Henrique Douglas Melo Coutinho ${ }^{1}$;Maria Flaviana Bezerra Morais-Braga ${ }^{1}$

\section{Resumo}

Leishmanioses e tripanossomíases são doenças parasitárias globalmente difundidas com elevado índice de mortalidade e morbidade. $\mathrm{O}$ arsenal terapêutico é restrito e de efeitos colaterais indesejáveis. No entanto, a biodiversidade de plantas brasileiras representa rica fonte de novos compostos com potencial antiparasitário. O geraniol é um monoterpeno encontrado em plantas como Cymbopogon winteranus e Cymbopogon martinii. Ocitronelal é um fitoconstituínte monoterpenóide de Eucalyptus citriodora e Cymbopogon citratus. O objetivo deste estudo foi avaliar a atividade antiparasitária in vitro desses terpenoides isolados contra formas epimastigotas e promastigotas de Trypanosoma cruzi e Leishmania. brasiliensis, bem como verificar sua citotoxicidade em células de mamíferos. As substâncias foram testadas nas concentrações de 50 e $100 \mu \mathrm{g} / \mathrm{mL}$, contudo, geraniol demonstrou baixa atividade contra $T$. cruzi, nestas concentrações, não demonstrando efeito contra promastigotas de L. braziliensis e revelando elevada citotoxicidade sobre células de mamíferos. O citronelal apresentou atividade moderada antipromastigota revelando altos índices de citotoxicidade.

Palavras-chave: Atividade antiparasitária; citototoxicidade; monoterpenos.

\section{ACTIVITY ASSESSMENT TRYPANOCIDAL, LEISHMANICIDE CYTOTOXIC AND THE GERANIOL AND CITRONELAL}

\begin{abstract}
Leishmaniasis and trypanosomiasis are globally widespread parasitic diseases with high mortality and morbidity. The therapeutic arsenal is restricted or undesirable side effects. However, the biodiversity of Brazilian plants is rich source of new compounds with potential antiparasitic. The monoterpene geraniol is found in plants such Cymbopogon winteranus and Cymbopogon martinii. Citronelal is a phytoconstituent monoterpenoid from Eucalyptus and Cymbopogon citratus. The objective of this study was to evaluate the in vitro antiparasitic activity of these terpenoids isolated against epimastigotes and promastigotes of Trypanosoma cruzi and Leishmania brasiliensis and verify their cytotoxicity in mammalian cells. The substances were tested at concentrations of 50 and $100 \mathrm{ug} / \mathrm{ml}$, however, geraniol showed low activity against $T$. cruzi in such concentrations that showed no effect against L. braziliensis promastigotes and showing high cytotoxicity to mammalian cells. Citronelal showed moderate activity antipromastigota revealing high levels of cytotoxicity.
\end{abstract}

Keywords: antiparasitic activity; cytototoxicity; monoterpenes.

\section{Introdução}

Doenças negligenciadas são aquelas causadas por agentes infecciosos ou parasitas consideradas endêmicas em regiões de baixa-renda, como na África, América Latina e Ásia. Por conta do baixo investimento em pesquisas, produção de medicamentos e controle, enfermidades como leishmaniose, doença de Chagas, dengue, malária, esquistossomose, tripanossomíase africana, tuberculose, dentre outras, continuam sendo uma das principais causas de mortalidade em todo mundo, levando a óbito cerca de 1 milhão de pessoas anualmente (AFN, 2013).

\footnotetext{
${ }^{1}$ Laboratory of Microbiology and Molecular Biology, Regional University of Cariri, Crato, Brazil

${ }^{2}$ Centre for the Development of Scientific la Investigación (CEDIC), Fundación Moisés Bertoni /Laboratorios Diaz Gill, Asuncion, Paraguay

*Autor correspondente:Maria Flaviana Bezerra Morais Braga. Departamento de Ciências Biológicas. Universidade Regional do Cariri - URCA, Crato (CE),

Brasil. Endereço: Rua Cel. Antônio Luís, 1161, Pimenta, CEP: 63105-00. Fone:+55 (88) 3102 1212; Fax: +55(88)31021291. E-mail:

flavianamoraisb@yahoo.com.br
} 
O agente causador da Doença de Chagas é o Tryponosoma cruzi, protozoário da família Trypanosomatidae pertencente à ordem Kinetoplastida, no qual seu ciclo de vida envolve obrigatoriamente a passagem por um hospedeiro intermediário - Triatoma infestans (barbeiro) para um definitivo, passando por uma série de estágios. Apresenta flagelo (epimastigota e tripomastigota) ou aflagelado (amastigota) dependendo do hospedeiro (ALBARACI, 2006). Por conta do considerado fluxo de pessoas em países como Estados Unidos, Canadá e Europa, a doença vem ganhando espaço e tem se tornado problema sério, nesses países (RASSI-JR, L.; RASSI, L.; MARIN-NETO, 2010).

A leishmaniose é uma doença que ameaça homens, mulheres e crianças ao redor do mundo, afetando aproximadamente 12 milhões de pessoas. A doença pode apresentar diversas manifestações clínicas, afetando a pele, mucosas e órgãos internos. A cutânea é forma mais comum, enquanto a visceral é considerada fatal, se não tratada. É causada por protozoários do gênero Leishmania, transmitidos pela picada de mosquitos flebótomos infectados (WHO, 2010). Como na doença de Chagas, os parasitas causadores da leishmaniose apresentam-se diversas formas durante seu ciclo de vida: amastigota, promastigota e paramastigota (NEVES et al., 2005).

Atualmente, dois medicamentos são usados para o tratamento da doença de Chagas, o benzonidazol e nifurtimox, porém o último citado é contra indicado em pacientes com antecedentes psiquiátricos ou distúrbios neurológicos (WHO, 2010). Tanto para tratamento das leishmanioses cutâneas e viscerais, os antimoniais pentavalentes têm sido utilizados como drogas de primeira escolha, apesar de sua alta toxicidade. A anfotericina B lipossomal, pentamidina, paramomicina e miltefosine são drogas de importância por representarem novas alternativas terapêuticas, porém apresentam amplos problemas como efeitos colaterais, preço do produto e produção da formulação (PEREIRA, 2011). Devido à dificuldade de encontrar novas drogas eficazes nessas terapias e que não sejam tóxicas para o hospedeiro, produtos naturais tem sido testados e investigados suas atividades leishmanicida e tripanocida (MESQUITA et al., 2005).

Óleos essenciais são produtos resultantes do metabolismo secundário das plantas, podendo ser extraídos por todos os órgãos, como brotos, folhas, flores, caules, galhos, raízes e sementes e são armazenados em células secretoras, epidérmicas e tricomas. Devido a sua rica composição química, dentre os principais são terpenoides, alcaloides e cumarinas, os óleos essenciais tem ganhado cada vez mais notoriedade e despertado interesse, dentre pesquisadores do mundo todo, devido as suas inúmeras propriedades (RESCHKE; MARQUES; MAYWORM, 2007; BAKALLI, 2008).

O geraniol (Fig.1 A), conhecido como rhodinol, é um álcool monoterpenoide, presente na constituição química de óleos essenciais extraídos de plantas como citronela e palmarosa. Por conta de seu odor é abundantemente empregado na fabricação de perfumes e como aromatizante de alimentos (BARNARD; XUE, 2004). Além disso, possui diversas propriedades farmacológicas dentre elas, podemos citar ação inseticida e repelente (TRAINA et al., 2005; JEON; LEE, C. H.; LEE, H. S., 2009; KHALLAAYPOUNE et al., 2009), efeito helmíntico (KURAMAN et al., 2003; HIERRO et al., 2004), antimicrobiano (FRIEDMAN et al., 2002; INOUYE et al., 2001; SI et al., 2006), antioxidante (CHOI et al., 2000; TIWARI, M.; KAKKAR, P., 2009) e atividade 
antitumoral in vitro e in vivo, contra leucemia, hepatoma e células de melanoma (SHOFF et al., 1991; CARNESECHI et al., 2001).

O citronelal (Fig.1 B), também pertencente ao grupo dos álcoois monoterpenoides e é o principal componente nas misturas de compostos químicos terpenoides, dando ao óleo de citronela o seu aroma característico de limão acentuado (JEONG-KYUet al.,2005). Sendo uma das substâncias majoritárias de óleos essenciais de plantas aromáticas (QUINTANS-JUNIOR et al., 2011), como as do Gênero Cymbopogon (BRITO et al., 2012; CAVALCANTI et al., 2011) e Eucalyptus (BRITO et al., 2012; TOMAZ et al., 2014) o citronelal revelou ter inúmeras atividades, dentre elas, podemos exemplificar ação antimicrobiana (SEIXAS et al., 2011; CAVALCANTI et al., 2011), alelopática (BRITO et al., 2012; TOMAZ et al., 2014), antioxidante (SCHERER et al.,2009; ANDRADE et al., 2012), herbicida (BRITO et al., 2012) e atividade inseticida e repelente (CHAGAS et al., 2002).

Esse estudo tem como objetivo avaliar a atividade antiparasitária in vitro de dois terpenóides isolados, o citronelal e o geraniol, geralmente encontrados em óleos essenciais, contra as formas epismastigotas e promastigotasde $T$. cruzi e $L$. brasiliensis, respectivamente, bem como verificar sua citotoxicidade em células de mamíferos.

Figura 1. Estrutura química dos monoterpenóides avaliados.

A<smiles>CC(C)=CCCC(C)=CCO</smiles>

B<smiles>CC(C)=CCCC(C)CC=O</smiles>

A: Geraniol; B: Citronelal

\section{Materiais e Métodos}

\section{Substâncias utilizadas}

As substâncias avaliadas foram o geraniol e citronelal fornecidas pela Empresa Sigma Chemical Co. (St. Louis, E. U. A). Como reagentes foram utilizados a Resazurina sódica obtida da Sigma-Aldrich (St Louis, MO) armazenada a $4{ }^{\circ} \mathrm{C}$ protegida da luz cuja solução de resazurina foi preparada em solução de fosfato $1 \%$ (PBS), $\mathrm{pH}$ 7, filtrada e esterilizada antes do uso; e o Clorofenol vermelho- $\beta$-D-galactopiranosídeo (CPRG; Roche, Indianápolis, IN) foi dissolvido em $0.9 \%$ Triton X- 100 (pH 7.4). Os antibióticos penicilina G (Ern, S.A., Barcelona, Espanha) e estreptomicina (Reig Jofr'e S.A., Barcelona, Espanha) foram adicionados para garantir ausência de crescimento bacteriano. 


\section{Teste de Citotoxicidade}

No ensaio de citotoxicidade foram utilizadas estirpes de fibroblastos NCTC 929 sendo cultivadas em meio Roswell Park Memorial Institute (RPMI) 1640 (SIGMA) suplementado a 10\% de soro fetal bovino (FBS) inativado pelo calor $\left(30\right.$ minutos a $\left.56^{\circ} \mathrm{C}\right)$, penicilina $\mathrm{G}(100 \mathrm{U} / \mathrm{mL})$ e estreptomicina $(100 \mathrm{mg} / \mathrm{mL})$. Para os experimentos, as células na fase pré-confluência foram colhidas com tripsina. Culturas de células foram mantidas a $37^{\circ} \mathrm{C}$ em uma atmosfera umidificada de $5 \%$ de $\mathrm{CO}_{2}$. O procedimento para a medição de viabilidade celular foi avaliada a partir do método de colorimetria com a metodologia proposta por Rólon et al. (2006).

As células clones foram semeadas $\left(3 \times 10^{4}\right)$ em placas de microdiluição de fundo chato de 96 poços, com $100 \mu \mathrm{L}$ de meio cultura RPMI 1640 por poço. As células foram cultivadas durante a noite a temperatura de $37^{\circ} \mathrm{C}$ e atmosfera de $5 \%$ de $\mathrm{CO}_{2}$. Em seguida o meio foi substituído pela substância isolada testada e adicionada em diferentes concentrações em $200 \mu \mathrm{L}$ do meio por 24 h. Foram incluídas poços como controles de crescimento. Após a incubação foi adicionado $20 \mu \mathrm{L}$ de solução resazurina a $2 \mathrm{mM}$ a cada poço. As placas foram incubadas novamente durante $3 \mathrm{~h}$; a redução da resazurina foi determinada por medida de absorbância do comprimento de onda a 490 e $595 \mathrm{~nm}$ em um leitor de microplacas.

Cada concentração foi testada três vezes. Percentagens de citotoxicidade $(\% \mathrm{C})$ dos produtos naturais foram determinadas da maneira como segue:

$\% \mathrm{C}=\left[\left(\mathrm{A}_{570} \times 117,216-\mathrm{A}_{595} \times 80,586\right)\right.$ de amostras de teste $/\left(\mathrm{A}_{570} \times 117,216-\mathrm{A}_{595} \times 80,586\right) \mathrm{de}$ controle)] x 100, onde, $A_{570}$ e $A_{595}$ representam os valores de meios de densidade óptica a 570 e $595 \mathrm{~nm}$, respectivamente, registrados para poços com células contendo diferentes doses do produto natural ou valor registrado para poços com células e sem produto natural (controla o crescimento positivo). Os valores 80,586 e 117,216 representam coeficientes de extinção molar para resazurina oxidada a $570 \mathrm{~nm}$ e $595 \mathrm{~nm}$, respectivamente.

\section{Atividade Leishmanicida e Tripanocida}

Para o ensaio de atividade leishmanicidain vitro, foram utilizadas formas promastigotas de L. braziliensis (MHOM/CO/88/UA301) a $26{ }^{\circ} \mathrm{C}$ em Schneider's (meio para inseto) suplementado a $10 \%$ (v/v) de SBF inativado pelo calor, $2 \%$ de urina humana normal (v/v) mais penicilina e estreptomicina. O método utilizado no ensaio foi desenvolvido por Mikus e Steverding (2000) modificado, onde as culturas promastigotas de L. brasiliensis $(2.5 \mathrm{x}$ $10^{5}$ parasitas/poço) foram cultivadas em placas de microdiluição. Tanto o geraniol e citronelal foram dissolvidos em dimetilsufóxido (DMSO). Foram realizadas diferentes diluições das substâncias de até $200 \mathrm{~mL}$ do volume final. Após a incubação de $48 \mathrm{~h}$ a $26{ }^{\circ} \mathrm{C}$, foi adicionado $20 \mu \mathrm{L}$ de solução de resazurina e a oxidação-redução foi quantificada a 570 a $595 \mathrm{~nm}$. Cada concentração foi testada em triplicata. Drogas de referência foram utilizadas como controle em cada ensaio. As porcentagens antipromastigotas (\%AP) formam calculadas, sendo a eficácia das substâncias testadas.

Para a avaliação tripanocida, foram utilizadas placas de microdiluição, contendo 96 poços (Sarstedt, Sarstedt, Inc.) com culturas que não atingiram a fase estacionária, de acordo com a descrição de Vega et al. (2005). 
As formas epimastigotas de $T$. cruzi foram semeadas a $1 \times 10^{5}$ por mililitro em $200 \mu \mathrm{L}$ e as placas foram incubadas com as substâncias testadas a $28{ }^{\circ} \mathrm{C}$ por 72 horas, foi adicionado $50 \mu \mathrm{L}$ de solução de CPRG a fim de dar a concentração final de $200 \mu \mathrm{M}$. As placas foram incubadas a temperatura de $37{ }^{\circ} \mathrm{C}$ por um período de $6 \mathrm{~h}$ adicionais e então lidas a $595 \mathrm{~nm}$ em espectrofotômetro.

A droga utilizada como referência foi o nifurtimox. Cada concentração foi testada em triplicata. Sendo cada experimento realizado duas vezes separadamente. O percentual de inibição (\%AE) foi calculado seguindo a fórmula: \% $\mathrm{AE}=\left[\left(\mathrm{AE} \_\mathrm{AEB}\right) /\left(\mathrm{AC} \_\mathrm{ACB}\right)\right] \times 100$, onde $\mathrm{AE}=$ absorvância do grupo experimental; $\mathrm{AEB}=$ branco de compostos; $\mathrm{AC}=$ grupo controle de absorvância; $\mathrm{ACB}=$ branco de meio de cultura. As soluções dos produtos naturais a serem analisadas foram preparadas em dimetilsufóxido, com a concentração final uma mistura água/DMSO jamais excedendo $0.2 \%$ do solvente final.

\section{Resultados e Discussão}

Os resultados deste trabalho mostraram que o citronelal, em ambas as concentrações testadas, apresentaram um percentual de 52,94\%, enquanto o geraniol apresentou um percentual de citotoxicidade de $27,06 \%$ e $32,94 \%$, nas concentrações de 100 e $50 \mu \mathrm{g} / \mathrm{mL}$ respectivamente, com relação à atividade antiepimastigota (Tabela 1). Verificou-se que a citotoxicidade se encontra em valores elevados de aproximadamente 40 a $85 \%$, respectivamente (Tabela 1), supondo-se que as amostras utilizadas nas concentrações de 100 e $50 \mu \mathrm{g} / \mathrm{mL}$ causam efeito biocida mais expressivo contra os fibroblastos do que frente às formas epimastigotas de T. cruzi.

$\mathrm{Na}$ avaliação da atividade antipromastigota, o citronelal apresentou um efeito intermediário tendo percentuais entre 62 e $65 \%$, entretanto sua toxicidade é elevada. O geraniol não demonstrou efeito sobre $L$. braziliensis (Tabela 1).

Tabela 1: Citotoxicidade, atividade antiepimastigota e antipromastigota do geraniol e citronelal.

\begin{tabular}{lllll}
\hline & \multicolumn{3}{c}{ Citronelal } & Geraniol \\
\cline { 2 - 5 } Concentrações & $100 \mu \mathrm{g} / \mathrm{mL}$ & $50 \mu \mathrm{g} / \mathrm{mL}$ & $100 \mu \mathrm{g} / \mathrm{mL}$ & $50 \mu \mathrm{g} / \mathrm{mL}$ \\
$\% \mathrm{AE}$ & 52,94 & 52,94 & 27,06 & 32,94 \\
$\% \mathrm{AP}$ & 64,42 & 62,92 & 0 & 0 \\
$\% \mathrm{C}$ & 84,07 & 55,31 & 41,50 & 45,63 \\
$\pm \% \mathrm{DS}$ & 5,39 & 4,08 & 5,39 & 3,53 \\
\hline
\end{tabular}

Conc.: concentração; \%C: percentual de citotoxicidade; \%AE: percentual de atividade antiepimastigota; \%AP: percentual de atividade antipromastigota; $\pm \%$ DS: Desvio Padrão.

A exposição de células de mamíferos a uma determinada substância, observando as mudanças causadas por ela, é a base do teste de citotoxicidade. As alterações acarretadas variam desde a lise celular, a inibição da formação de colônias celulares, deslocamento dos tecidos entre outros (ROGERO et al., 2003). Os fibroblastos por serem 
células de fácil manutenção e estarem presentes em ferimentos, contribuindo intensamente para regeneração do tecido conjuntivo, são escolhidos em grande escala para realização de testes de citotoxicidade (RATNER et al., 2004).

Estudos mostraram que o geraniol demonstrou atividade antiparasitária contra Leishmania infantum (MORALES et al., 2009). A inexistência da atividade leishmanicida nos testes com geraniol contra L. braziliensis, diferenciando-se dos resultados obtidos por Morales et al. (2009), pode ser atribuída às alterações nas sequências genéticas, responsáveis por promoverem a variabilidade entre as espécies e consequentemente determinarem fenotipicamente a resistência destes organismos, que acaba repercutindo na ineficácia da bioatividade de alguns compostos. Estes são testes preliminares podendo revelar um potencial antiparasitário, direcionando de forma segura pesquisas in vivo.

As análises dos fatores bioquímicos mostraram que tanto Leishmania e Trypanosoma demonstraram vias metabólicas semelhantes a células hospedeiras, por isso o tratamento para as doenças causadas por esses protozoários é muito difícil. (SOARES-BEZERRA et al., 2004). Assim, a pesquisa por novos medicamentos com atividade antiparasitária e baixa toxicidade é muito importante, para serem usados como fontes terapêuticas no combate a tais enfermidades.

\section{Conclusões}

O geraniol apresentou uma baixa atividade contra T. cruzi, em ambas as concentrações e não demonstrou efeito contra as formas promastigotas de L. braziliensis. O citronelal demonstrou uma atividade antipromastigota moderada e baixo potencial antiparasitário contra $T$. cruzi. Em relação à citotoxicidade em células de mamíferos, ambos os monoterpenos revelaram significativo potencial citotóxico quando comparado ao efeito verificado contra as formas parasitárias.

\section{Referências}

AGÊNCIA FIO CRUZ DE NOTICIAS - AFN. 2013. Cidade: Manguinhos. Disponivel em: http://www.agencia.fiocruz.br/doen\%C3\%A7as-negligenciadas. Acesso: em 14 de Novembro de 2014.

ALBARACI, T. R. Estudo fitoquimico de Rauiasp. Econchocarpus macrophyllus (Rutaceaea) e avaliação da atividade antiparasitária de extratos e substâncias isoladas. 2006. Tese (Doutorado em Química Orgânica) Universidade Federal de São Carlos, São Carlos, SP, 2006.

ANDRADE, M.A. et al. Óleos essenciais de Cymbopogon nardus, Cinnamomum zeylanicum e Zingiber officinale: composição, atividades antioxidante e antibacteriana. Revista Ciência Agronômica, v. 43, n. 2, p. 399-408, 2012.

BAKALLI, F. et al. Biological effects of Essentials oils - a review. Food and Chemical Toxicology, Oxford, v. 36, n.2, p. 446-475, 2008. 
BARNARD, D. R.; XUE, R. Laboratory evaluation of mosquito repellents against Aedes albopictus, Culex nigripalpus and Ochlerotatus triseriatus (Diptera: Culicidae). Journal of Medical Entomology, v. 41, n. 4, p. 726730, 2004.

BRITO, D.V. et al. Effect of citronella oil, eucalipto and citronellal compound of mycoflora and devolopment of maize plants. Journal of Biotechonology and Biodiversity, v.3, p. 184-192, 2012.

CARNESECHI, S. et al. Geraniol, a component of plant essential oils, inhibits a growth and polyamine biosynthesis in human colon cancer cells. The Journal of Pharmacology and experimental therapeutics, v. 298, n.1, p. 197-200, 2001.

CAVALCANTI, Y. M.; ALMEIDA, L.F.D.; PADILHA, W.W.N. Screening of essential oils antifungal activity on Candida strains. Odontologia Clínica-Científica, Recife, p. 243-246, 2011.

CHAGAS, A. C. S. et al.Efeito acaricida de óleos essenciais e concentrados emulsionáveis de Eucalyptus spp em Boophilus microplus. Brazilian Journal of Veterinary Research and Animal Science, São Paulo, v. 39, p. 247253, 2002.

CHOI, H. S. Radical-scavenging activities of citrus essential oils and their components: detection using $1,1-$ diphenyl-2-picrylhydrazl. Journal of Agriculture and Food Chemistry, v. 48, p. 4156-4161, 2000.

FRIEDMAN, M.; HENIKA, P. R; MANDRELL, R. E. Bactericidal activities of plant esential oils and some of their isolated constituents against Campylobacer jejuni, Escherichia coli, Listeria monocytogenes and Salmonella enterica. Journal of Food Protection, v. 10, p. 1545-1560, 2002.

HIERRO, I. Action of different monoterpenic compounds against Anisakis simplex s. I. L3 larvae. Phytomedicine, v. 11, p.77-82, 2004.

INOUYE, S.; TAKIZAWA, T.; YAMAGUCHI, H. Antibacterial activity of essential oils and their major constituents against respiratory tract pathogens by gaseous contact.Journal of Antimicrobial Chemotherapy, v. 47, p. 565-573, 2001.

JEON, J. H.; LEE, C. H.; LEE, H. S. Food protective effect of geraniol and its congeners against stored food mites. Journal of Food Protection, v. 72, p.1468-1471, 2009.

JEONG-KYU, K. et al. Evaluation of Repellency Effect of Two Natural Aroma Mosquito Repellent Compunds Citronella and Citronellal, Entomological Reserach, v. 35, n.2, 117-120, 2005.

KHALLAAYOUNE, K. et al. Efficacy of 1\% geraniol (Fultec) as a tick repellent. Parasite, v.16, p.223-226, 2009.

KURAMAN, A. M. et al. Geraniol, the putative anthelmintic principle of Cymbopogon martinii. Phototherapy Research, v. 17, p. 957, 2003.

MESQUITA, M. L. et al. Antileishmanial and trypanocidal activity of Brazilian Cerrado plants. Memórias do Instituto Oswaldo Cruz, v. 100, n. 7, p. 783-787, 2005.

MIKUS J.; STEVERDING, D.A simple colorimetric method to screen drug cytotoxixity against Leishmania using the dye Alarmar Blue. Parasitol International, v.48, p. 265-269, 2000.

MORALES, M. et al. Activity of different monoterpenic derivates of natural origen against Leishmania infantum promastigotas. Revista Ibero-latino americana de Parasitologia, v.1: p. 65-72, 2009.

NEVES, D. P. et al. Parasitologia Humana. 11 ed. São Paulo, p.47-64, 2005.

PEREIRA, IO.; SACRAMENTO L.V.S.; MARQUES, M.J.; Leishmanioses: o estado da arte. Revista da Universidade Vale do Rio Verde. v.9, n.1, p.220-38, 2011. 
QUITANS-JUNIOR, L. et al. Antinociceptive action and redox properties of citronelal, an essential oil present in lemon grass. Journal of Medicinal Food, v.14, n.6, p.630-639, 2011.

RASSI-JR, A.; RASSI, A.; MARIN-NETO, J.A. Chagas disease. PubMed, v. 375, n. 9723, p.1388-402, 2010.

RATNER, B. et al. Biomaterials science: an introduction to materials in medicine. SecondEdition, ElsevierAcademic Press, 2004.

RESCHKE, A.; MARQUES, L.M.; MAYFORM, M.A.S. Atividade antibacteriana de Ficus benjamina L. (Moraceae) Revista Brasileira de Plantas Medicinais: brazilian jornal of medicinal plants, Botucatu, v. 9, n. 2, p. 67-70, 2007.

ROGERO, S. O. et al. Teste in vitro de citotoxicidade: estudo comparativo entre duas metodologias. Materials Research. São Carlos, v. 6, n. 3, p. 317-320, 2003.

ROLÓN, M. et al. Selective activity of polyene macrolides produced by genetically modified Streptomyces on Trypanosoma cruzi. International Journal of Antimicrobial Agents, v. 28, n. 2, p. 104 -109, 2006.

SCHERER, R. et al. Composição e atividades antioxidante e antimicrobiana dos óleos essenciais de cravo-da-india, citronela e palmarosa. Revista Brasileira de Plantas Medicinais, Botucatu, v.11, n.4, p.442-449, 2009.

SEIXAS, T.L.; CASTRO, H.C.; SANTOS, G.R.; CARDOSO, D.P.; Controle fitopatológico do Fusarium subglutinans pelo óleo essencial do capim-citronela (Cymbopogon nardus L.) e do composto citronelal. Revista Brasileira de Plantas Medicinais, Botucatu, v. 13, Especial, pp. 523-526, 2011.

SHOFF, S. M. et al. Concentration- dependent increase of murine P388 and B16 population doubling time by the acyclic monoterpene geraniol. Cancer Research, v.51,p. 37- 42, 1991.

SI, W. et al. Antimicrobial activity of essential oils and structurally related synthetic food additives selected pathogenic and beneficial gut bacteria. Journal of Applied Microbiology, v.100, p. 296-305, 2006.

SOARES-BEZERRA, R.J.; LEON, L.; GENESTRA, M.M. Recentes avanços da quimioterapia das leishmanioses: moléculas intracelulares como alvo de fármacos. Brazilian Journal of Pharmaceutical Science,v.40, p. 139-149, 2004.

TIWARI, M.; KAKKAR, P. Plant derived antioxidants-geraniol and camphene protect rat alveolar macrophages against t-BHP induced oxidate stress. Toxicology in Vitro, v.23, p. 295-301, 2009.

TOMAZ, M.A. et al. Chemical Composition and Allelopathic Activity of the Eucalyptus Essential Oil. Bioscience Journal, Uberlândia, v. 30, p. 475-483, 2014.

TRAINA, O. et al. In vitro acaricidal activity of four monoterpenes and solvents against Otodectes cynotis (Acari: Psoroptidae). Experimental and Applied Acarology, v.37,p. 141-146, 2005.

VEGA, C. et al. A new pharmacological screening assay with Trypanosoma cruzi epimastigotas expressing betagalactosidase. Parasitol Research, v. 95, p. 296-298, 2005.

WORLD HEALTH ORGANIZATION. 2010. Working to overcome the global impact of neglected tropical diseases: First WHO report on neglected tropical diseases. - WHO. Disponível em:http://www.who.int/neglected diseases/2010report/NTD 2010report embargoed.pdf _Acesso: em 14 de Novembro de 2014.

Recebido: $13 / 02 / 2015$

Aceito: $22 / 02 / 2015$ 